\title{
Application of Percutaneous Coronary Sinus Occlusion in Patients with Acute Coronary Syndrome Without Hemodynamically Significant Atherosclerotic Coronary Lesions
}

DOI: $10.17691 / \mathrm{stm} 2016.8 .1 .02$

Received September 9, 2015

E.B. Shakhov, MD, PhD, Assistant, Department of Oncology, Postgraduate Faculty

Nizhny Novgorod State Medical Academy, 10/1 Minin and Pozharsky Square, Nizhny Novgorod, 603005, Russian Federation

Selective coronary angiography may reveal slightly affected coronary arteries with less than $50 \%$ of stenotic areas in $10-15 \%$ of patients with clinical and ECG picture of acute coronary syndrome.

The aim of the investigation is to evaluate the efficacy of the novel technique of endocardial and epicardial coronary bloodstream correction - intermittent percutaneous coronary sinus occlusion (PCSO) — in patients with acute coronary syndrome without hemodynamically significant atherosclerotic coronary lesions by studying the main functional parameters of the left ventricular myocardium in the medium terms (12 months later).

Materials and Methods. The results of treating 8 patients with acute coronary syndrome and ST-segment depression without hemodynamically significant coronary stenoses and initial diastolic dysfunction have been analyzed. Four patients underwent PCSO with subsequent medication, other four patients received only therapeutic treatment.

Results. In group 1 the myocardium function was observed to normalize completely in 2 individuals 12 months after the surgery (medium term). In group 2 complete normalization of diastolic myocardial function was not observed in any patient. Patients after PCSO showed statistically significantly better parameters of diastolic function 1 year after the operation compared to the results of only therapeutic treatment.

Conclusion. PCSO procedure combined with subsequent adequate therapeutic support in patients with acute coronary syndrome has significantly better effect on restoration completeness of left ventricular diastolic function compared to isolated therapeutic treatment.

Key words: acute coronary syndrome; native coronary arteries; intermittent percutaneous coronary sinus occlusion; myocardial function; echocardiography parameters.

Developing new concepts and improving treatment methods for patients with acute coronary syndrome (ACS) contributes to accumulation of information on the possibility of myocardium ischemia without hemodynamically significant atherosclerotic coronary lesions. The studies carried out in different ethnic groups show that selective coronary angiography (SCA) can reveal slightly affected coronary arteries with less than $50 \%$ of stenosis segments in $10-15 \%$ of patients with clinical and electrocardiographic picture of ACS $[1,2]$. This paradox is associated with the phenomena of distal coronary artery spasm leading to reduced peripheral myocardial perfusion and, consequently, to compromised cardiac muscle perfusion [3].

The method of intermittent percutaneous coronary sinus occlusion (PCSO) offered by van de Hoef et al. [4] proved to be effective in restoration of adequate epicardial and endocardial coronary bloodstream in ACS patients with ST-elevation after stenting infarctionassociated coronary system. However, the expedience of PCSO application in patients with acute myocardium ischemia without hemodynamically significant atherosclerotic coronary lesions as well as the influence of temporary coronary sinus occlusion on the left ventricular (LV) function have been a scientific problem studied insufficiently.

The aim of the investigation is to evaluate the efficacy of the novel technique of endocardial and epicardial coronary bloodstream correction intermittent percutaneous coronary sinus occlusion in patients with acute coronary syndrome without hemodynamically significant atherosclerotic coronary lesions by analyzing the main functional parameters of the left ventricular myocardium in the medium terms (12 months later).

Materials and Methods. The results of treating 8 patients with ACS non-ST-elevation at the age of 38 to 73 years (average age $56.7 \pm 12.6$ years) staying in City Clinical Hospital No.5 of Nizhny Novgorod (Russia) were analyzed from October 9, 2014 till February 2, 2015. No one of the examined patients had reliable coronary past history (Table 1 ).

For contacts: Evgeniy B. Shakhov, e-mail: es-ngma@yandex.ru 
Table 1

\section{Clinical characteristics of patients with acute coronary syndrome}

\begin{tabular}{lc}
\hline \multicolumn{1}{c}{ Anamnestic features } & $\begin{array}{c}\text { Quantity } \\
\text { of patients } \\
\text { (abs. number/\%) }\end{array}$ \\
\hline $\begin{array}{l}\text { Gender differences: } \\
\text { males }\end{array}$ & $4 / 50$ \\
females & $4 / 50$ \\
\hline Pain syndrome characteristics: & \\
newly diagnosed angina pectoris, FC III, & \\
according to CCS & $8 / 100$ \\
post-stress angina pectoris & $5 / 63$ \\
exercise-induced angina pectoris & $3 / 37$ \\
Risk factors for coronary heart disease: & \\
smoking & $3 / 37$ \\
arterial hypertension, stage II, risk 2 & $6 / 75$ \\
genetic predisposition & $0 / 0$ \\
hyperlipidemia & $2 / 25$ \\
diabetes mellitus & $0 / 0$ \\
history of myocardial infarctions & $0 / 0$ \\
previous hospitalization for IHD & $0 / 0$ \\
\hline Pre-hospital medication therapy with: & \\
nitrates & $0 / 0$ \\
beta-blockers & $0 / 0$ \\
calcium antagonists & $0 / 0$ \\
aspirin & $0 / 0$ \\
hypolipidemic agents & $0 / 0$ \\
\hline
\end{tabular}

The study complies with the Declaration of Helsinki (adopted in June 1964 (Helsinki, Finland) and revised in October 2000 (Edinburgh, Scotland)) and approved by the Ethics Committee of Nizhny Novgorod State Medical Academy. Written informed consent was obtained from every patient.

The study included only the patients whose ECG presented evidence of acute myocardial ischemia in the "anterior" group of thoracic leads $\left(\mathrm{V}_{1}-\mathrm{V}_{3}\right)$ on hospital admission despite intensive medication treatment with mandatory antianginal and antithrombotic therapy at the pre-hospital stage.

In all cases the patients underwent pre-hospital analysis of fatal outcome and myocardial infarction risks $[5,6]$, which determined the selection of management strategy for ACS patients in hospital. In case of high risk of lethal outcome or cardiac muscle infarction as well as persistent ischemic changes in $V_{1}-V_{3}$ leads on electrocardiogram such patients underwent SCA in the first $24 \mathrm{~h}$ of hospital admission, according to the concept of early invasive strategy.

We focused on the study of distal coronary spasm accompanied by reduced peripheral myocardial perfusion and slowing antegrade coronary blood flow, mainly in the vascular territory of the anterior descending artery (ADA). ACS patients without angiographically documented damage of endocardial and epicardial coronary blood flow were not included in the study.

An angiographic criterion of inclusion of the examined patients in the investigation was absence of hemodynamically significant damage in the vascular territories of the ADA, the circumflex artery (CA) and the right coronary artery (RCA) (Figure 1).

Stenoses to less than $50 \%$ were considered hemodynamically insignificant constrictions. Coronary lesions were distributed among the selected patients in the following way:

ADA stenoses no more than $20 \%-2(22.2 \%)$;

RCA stenoses no more than $20 \%-3(33.3 \%)$;

CA stenoses no more than $20 \%-1(11.1 \%)$;

no coronary arterial stenoses - $3(33.4 \%)$.

On performing SCA all patients under study were conventionally divided in two groups of four individuals.

Group 1 comprised patients with ACS who underwent SCA followed by PCSO which was performed according to the method developed by van de Hoef et al. [4]. The procedure of endovascular correction of peripheral
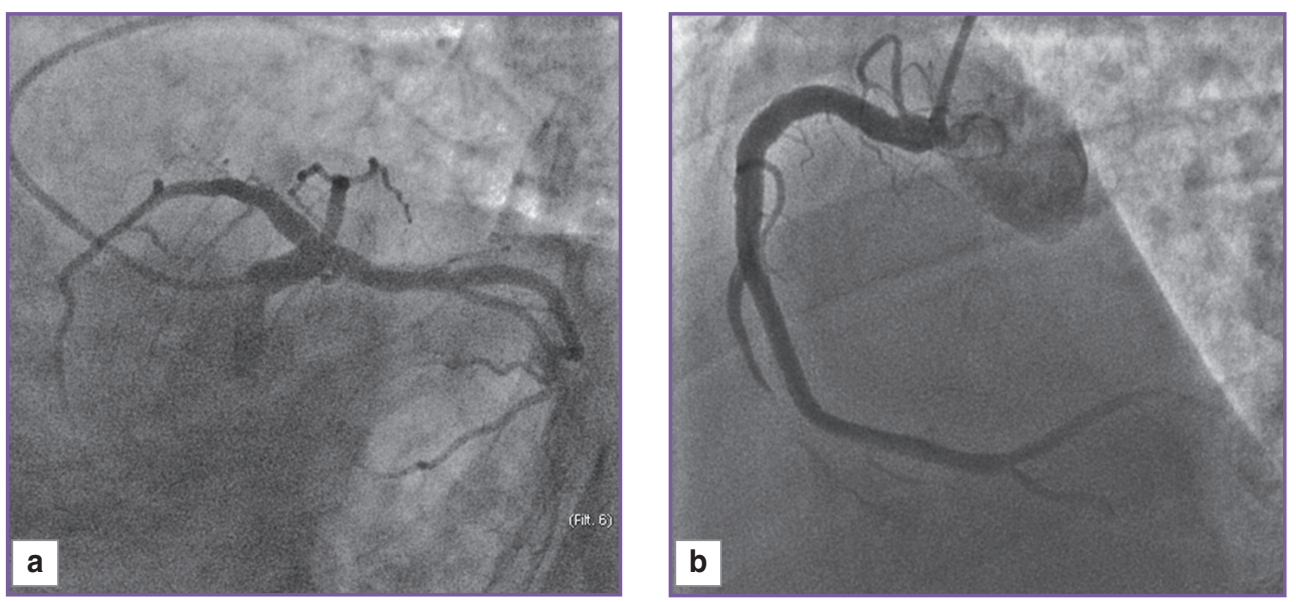

Figure 1. Absence of hemodynamically significant atherosclerotic coronary lesion on selective coronary angiogram: (a) vascular territories of the anterior descending artery and the circumflex artery; (b) vascular territory of the right coronary artery 

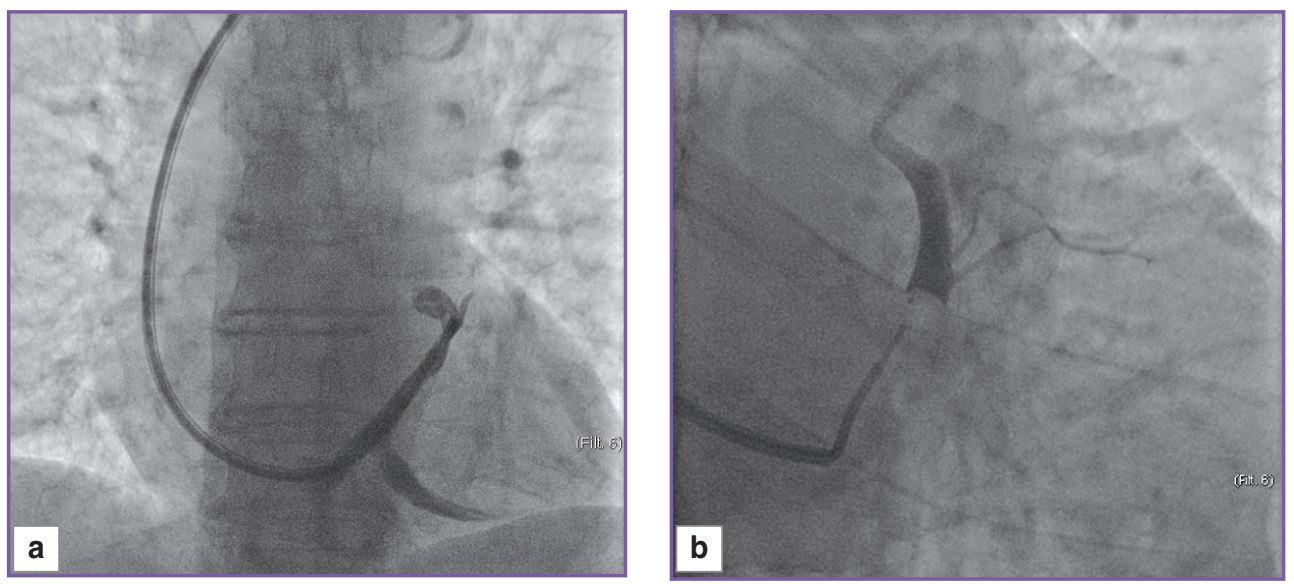

Figure 2. Stages of performing temporary occlusion of venous outflow from the myocardium: (a) positioning the delivery system; (b) occlusion of the main cardiac vein

myocardial perfusion in patients with acute myocardial ischemia and hemodynamically insignificant lesion of coronary arteries was carried out in our study for the first time.

To block the venous outflow from the myocardium temporarily the cardiac venous system was accessed by puncturing the right subclavian vein and installing a delivery system in the orifice of the coronary sinus (Figure 2).

The standard dual-lumen balloon catheter SwanGanz 7F was placed in the proximal part of the main cardiac vein over the delivery system. The balloon catheter was thoroughly positioned near the orifice of the coronary sinus before the small cardiac vein inflow. In such a position the catheter completely stopped the outflow through the main cardiac vein to the right atrium when the end balloon was maximally dilated (Figure 2 (b)). The free inner lumen of the balloon catheter was connected to the invasive pressure probe included in the diagnostic complex GE Healthcare Mac-Lab/SpecialsLab 6.8 (GE Medical Systems, USA). Catheterization of the cardiac venous system and positioning of the balloon catheter lasted $13.0 \pm 1.9$ min. Intermittent PCSO was performed for $10.4 \pm 1.5 \mathrm{~min}$ using the balloon catheter in the short-term "inflation-deflation" mode followed by obligatory control angiography 3 min after completion of the procedure.

On performing PCSO all patients of this group underwent obligatory therapeutic treatment with administration of calcium antagonists.

Group 2 included patients who underwent SCA without PCSO procedure. All of them also received medication treatment with calcium antagonists to manage distal coronary spasm in order to increase peripheral myocardial perfusion and indirectly improve the antegrade coronary blood flow.

To evaluate the efficacy of endovascular correction of peripheral myocardial perfusion in patients with ACS the analysis of the main echocardiographic (echoCG) parameters was performed in both groups during hospitalization as well as in the medium terms, 12 months after PCSO procedure and medication treatment (group 1) or only medication (group 2). Ultrasound scanner Vivid 7 Pro (GE Medical Systems, Norway) with $3.0-3.5 \mathrm{MHz}$ transducers, 2D-mode and Doppler imaging was used to perform echocardiography. During examination great attention was given to LV function parameters: ejection fraction (EF), impaired local contractility (ILC), early-tolate left ventricular filling ratio $(E / A)$.

Statistical data processing was performed by means of the program Statistica 8.0. The results were presented as mean \pm standard deviation $(\mathrm{M} \pm \mathrm{sd})$. Nonparametric statistical analysis of the obtained data by means of Wilcoxon paired signed rank test was used to compare two dependent variables (within-group analysis), and Mann-Whitney U-test to compare two independent variables (between-group analysis). Values at $p \leqslant 0.05$ were considered statistically significant [7].

Results. While studying the echoCG picture in ACS patients of both groups on hospital admission we paid attention to the following fact: despite the present ECG signs of acute myocardial ischemia on the anterior LV wall there was no evidence of impaired systolic and

Table 2

Initial basic functional parameters of the left ventricular myocardium in patients of both groups

\begin{tabular}{|c|c|c|c|}
\hline Parameters & Group $1(n=4)$ & Group $2(n=4)$ & p \\
\hline $\mathrm{LV} \mathrm{EF}_{\text {mean }}(\%)$ & $54.0 \pm 2.9$ & $57.0 \pm 4.7$ & 0.312 \\
\hline$I \mathrm{IC}_{\text {mean }}$ & $1.0 \pm 0.0$ & $1.0 \pm 0.0$ & - \\
\hline$E / A_{\text {mean }}$ & $0.65 \pm 0.07$ & $0.70 \pm 0.08$ & 0.248 \\
\hline
\end{tabular}

$\mathrm{N}$ o t e. $L V \mathrm{EF}_{\text {mean }}$ : left ventricular ejection fraction (mean value); $\mathrm{ILC}_{\text {mean }}$ : impaired local contractility (mean value); $E / A_{\text {mean }}$ : indicator of diastolic function (mean value). 
Table 3

Basic functional parameters of the left ventricular myocardium in patients with acute coronary syndrome before and 1 year after the intervention

\begin{tabular}{|c|c|c|c|c|c|}
\hline \multirow{2}{*}{ Parameters } & \multicolumn{2}{|c|}{ Group 1 (PCSO and therapy ) } & \multicolumn{2}{|c|}{ Group 2 (only therapy) } & \multirow{2}{*}{$p_{1-2}$} \\
\hline & Initial & 1 year after & Initial & 1 year after & \\
\hline \multirow{2}{*}{$\mathrm{LV} \mathrm{EF}_{\text {mean }}(\%)$} & $54.0 \pm 2.9$ & $65.0 \pm 4.4$ & $57.0 \pm 4.7$ & $66.5 \pm 4.2$ & \multirow{2}{*}{0.563} \\
\hline & \multicolumn{2}{|c|}{$p=0.067$} & \multicolumn{2}{|c|}{$p=0.067$} & \\
\hline \multirow{2}{*}{$I L C_{\text {mean }}$} & $1.00 \pm 0.0$ & $1.00 \pm 0.0$ & $1.00 \pm 0.0$ & $1.00 \pm 0.0$ & \multirow[b]{2}{*}{ 一 } \\
\hline & & & & & \\
\hline \multirow{2}{*}{$E / A_{\text {mean }}$} & $0.65 \pm 0.07$ & $1.04 \pm 0.14$ & $0.70 \pm 0.08$ & $0.80 \pm 0.09$ & \multirow{2}{*}{0.02} \\
\hline & \multicolumn{2}{|c|}{$p=0.067$} & \multicolumn{2}{|c|}{$p=0.067$} & \\
\hline
\end{tabular}

$\mathrm{N}$ o t e. $\mathrm{LV} \mathrm{EF}_{\text {mean: }}$ left ventricular ejection fraction (mean value); ILC $\mathrm{C}_{\text {mean: }}$ impaired local contractility (mean value); $E / A_{\text {mean }}$ indicator of diastolic function (mean value); PCSO: percutaneous coronary sinus occlusion.

myocardial contractile functions. However, according to echoCG data, all studied patients in both groups were found to have diastolic LV dysfunction of type 1 (impaired relaxation) (Table 2).

Within the framework of this investigation the patients of group 1 successfully underwent SCA combined with PCSO in the first $24 \mathrm{~h}$ of hospital admission, and isolated SCA was performed in the patients of group 2. Survival in both groups in early (within 12 months) and medium (after 12 months) control terms after the operation amounted to $100 \%$. Clinically and hemodynamically insignificant complication occurred during catheterization of the coronary sinus only in one patient of group 1 and it was associated with insignificant extravasation of contrast medium while performing coronary phlebography to position the dual-lumen balloon catheter. There was no perforation of the main cardiac vein during PCSO in any case.

During follow-up period 12 months after PCSO and medication treatment two patients of group 1 showed tendency to restore the diastolic function $\left(E / A_{\text {mean }}=0.95 \pm 0.03\right)$, and in two cases normalization of $E / A$ parameter $\left(E / A_{\text {mean }}=1.14 \pm 0.16\right)$ was observed. Improvement of systolic function (within the norm) occurred in all the 4 patients of group 1 $\left(\mathrm{EF}_{\text {mean }}=65.0 \pm 4.4 \%\right)$. Impaired local contractility was not revealed in any case $\left(\mathrm{ILC}_{\text {mean }}=1.00 \pm 0.0\right)$ (Table 3).

During the follow-up period 12 months after PCSO and medication treatment no one of the patients in group 2 showed normalization of $E / A$ parameter $\left(E / A_{\text {mean }}=0.80 \pm 0.09\right)$. Tendency for improvement of systolic function (within the norm) was observed in all the 4 patients under study $\left(\mathrm{EF}_{\text {mean }}=66.5 \pm 4.2 \%\right)$. There was no impaired local contractility found in any case $\left(\mathrm{ILC}_{\text {mean }}=1.00 \pm 0.0\right)$ (Table 3).

Analyzing echoCG dynamics in the studied patients with ACS in medium terms after hospitalization we paid special attention to the following fact: 12 months after PCSO the patients in group 1 showed definitely better diastolic function parameters compared to the patients of group 2 who were instituted only therapeutic treatment in order to correct epicardial and endocardial coronary blood flow $(p=0.20)$. No statistically significant between-group differences in systolic function and local contractility parameters analyzed at control echoCG examination were found $(p=0.563)$.

Discussion. At present the method of intermittent PCSO is one of the most challenging approaches to retrograde perfusion of cardiac venous system [8]. This procedure was implemented in clinical practice by virtue of investigations carried out by van de Hoef et al. (2012) who demonstrated effective temporary occlusion of the cardiac venous outflow with correction of peripheral myocardial perfusion in ACS patients with ST-elevation after stenting infarction-dependent ADA $[4,8,9]$. Changes in cardiohemodynamics of patients with ACS after PCSO procedure shown by the scientists were taken as the basis for application of the novel technique in patients with acute myocardial ischemia and hemodynamically insignificant atherosclerotic coronary lesions. Such approach to performing PCSO has been demonstrated in our study for the first time.

ECG picture of all studied patients without hemodynamically significant atherosclerotic coronary lesions at pre-hospital and hospital stage revealed ACS with persisting ischemic changes in $V_{1}-V_{3}$ leads despite intensive medication with obligatory administration of antianginal and anti-thrombotic therapy. Besides, there was no initial impairment of systolic function and local LV contractility in any case, while echoCG picture showed diastolic LV dysfunction of type 1 (impaired relaxation) in $100 \%$ of patients under study (in group $1-E / A_{\text {mean }}=0.65 \pm 0.07$, in group $2-$ $\left.E / A_{\text {mean }}=0.70 \pm 0.08 ; p=0.248\right)$. According to Rhew et al. [10], such discrepancy between angiographic, ECG picture and echoCG results is caused by positive influence of therapeutic treatment at pre-hospital stage, which reduces intensity of coronary spasm, hypercoagulability, microvascular embolism, myocardial inflammation. As a result, such patients (which was also 
observed in our case) develop no significant cardiac muscle damages leading to impairment of systolic function and local LV contractility. However, absence of complete medication-based restoration of adequate myocardial perfusion confirmed during SCA brings about the most sensitive echoCG marker of ischemia diastolic LV dysfunction [11].

In our study we revealed no significant withingroup and between-group differences in $\mathrm{LV} \mathrm{EF}_{\text {mean }}$ and ILC mean parameters in medium terms after the operative intervention. Yet the patients who underwent PCSO with subsequent medication treatment had significantly better diastolic function parameters a year after hospitalization for ACS as compared to those from "therapeutic" group $\left(E / A_{\text {mean }}\right.$ in group $1-1.04 \pm 0.14$, in group $2-$ $0.80 \pm 0.09 ; p=0.020)$. Such observation can be explained by investigations of the authors $[12,13]$ who proved that more significant improvement of diastolic function occurs in patients with myocardium ischemia when timely and maximally complete restoration of epicardial and endocardial blood flow velocity, mainly in the vascular territory of the ADA, is provided. Correction of antegrade coronary blood flow and peripheral myocardial perfusion contributes to stabilization of cardiac muscle cell metabolism promoting normalization of passive myocardial properties, cardiomyocyte relaxation velocity in particular, and restoration of diastolic LV function in general $[14,15]$. Precisely that was the reason why we observed diastolic function to normalize in 2 out of 4 patients of group 1. On the contrary, restoration of E/A parameter was not registered in any case in group 2 $\left(E / A_{\text {mean }}=0.80 \pm 0.09\right)$.

Conclusion. In case of hemodynamically insignificant atherosclerotic coronary lesion combined with clinical and ECG picture of acute coronary syndrome it is reasonable to perform intermittent percutaneous coronary sinus occlusion in order to correct myocardial coronary blood flow.

Temporary occlusion of myocardial venous outflow supplemented by adequate medication therapy has no statistically significant advantages in comparison with isolated therapeutic treatment as to rapidity and completeness of left ventricular systolic function restoration, however, it has significantly better effect on completeness of left ventricular diastolic function restoration.

Study Funding and Conflicts of Interest. This study was not funded by any sources, and there is no conflicts of interest associated with this study.

\section{References}

1. Moiseenkov G.V., Gayfulin R.A., Barbarash O.L., Berns S.A., Barbash L.S. "Clean" coronary arteries in patients with acute coronary syndrome. Mezhdunarodnyy zhurnal interventsionnoy kardioangiologii 2008; 14: 70a-71.

2. von Korn H., Graefe V., Ohlow M.A., Yu J., Huegl B., Wagner A., Gruene S., Lauer B. Acute coronary syndrome without significant stenosis on angiography. Tex Heart Inst J 2008; 35(4): 406-412.

3. Ong P., Athanasiadis A., Hill S., Vogelsberg H., Voehringer M., Sechtem U. Coronary artery spasm as a frequent cause of acute coronary syndrome: the CASPAR (coronary artery spasm in patients with acute coronary syndrome) study. J Am Coll Cardiol 2008; 52(7): 528-530, http://dx.doi. org/10.1016/j.jacc.2008.04.050.

4. van de Hoef T.P., Nolte F., Delewi R., Henriques J.P., Spaan J.A., Tijssen J.G., Siebes M., Wykrzykowska J.J., Stone G.W., Piek J.J. Intracoronary hemodynamic effects of pressure-controlled intermittent coronary sinus occlusion (PICSO): results from the First-In-Man Prepare PICSO Study. J Interv Cardiol 2012; 25(6): 549-556, http://dx.doi.org/10.1111/ j.1540-8183.2012.00768.x.

5. Antman E.M., Cohen M., Bernink P.J., McCabe C.H., Horacek T., Papuchis G., Mautner B., Corbalan R., Radley D., Braunwald $\mathrm{E}$. The TIMI risk score for unstable angina/non-ST elevation Ml: a method for prognostication and therapeutic decision making. JAMA 2000; 284(7): 835-842, http://dx.doi. org/10.1001/jama.284.7.835.

6. Tang E.W., Wong C.K., Herbison P. Global Registry of Acute Coronary Events (GRACE) hospital discharge risk score accurately predicts long-term mortality post acute coronary syndrome. Am Heart J 2007; 153(1): 29-35, http://dx.doi. org/10.1016/j.ahj.2006.10.004.

7. Petrov V.I., Nedogoda S.V. Meditsina, osnovannaya na dokazatel'stvakh [Medicine based on evidence]. Moscow: GEOTAR-Media; 2009.

8. Mohl W., Gangl C., Jusić A., Aschacher T., De Jonge M., Rattay F. PICSO: from myocardial salvage to tissue regeneration. Cardiovasc Revasc Med 2015; 16(1): 36-46, http://dx.doi. org/10.1016/j.carrev.2014.12.004.

9. van de Hoef T.P., Nijveldt R., van der Ent M., Neunteufl T., Meuwissen M., Khattab A., Berger R., Kuijt W.J., Wykrzykowska J., Tijssen J.G., van Rossum A.C., Stone G.W., Piek J.J. Pressure-controlled intermittent coronary sinus occlusion (PICSO) in acute ST-segment elevation myocardial infarction: results of the prepare RAMSES safety and feasibility study. Eurolntervention 2015; 11(1): 37-44, http:// dx.doi.org/10.4244/EIJY15M03_10.

10. Rhew S.H., Ahn Y., Kim M.C., Jang S.Y., Cho K.H., Hwang S.H., Lee M.G., Ko J.S., Park K.H., Sim D.S., Yoon N.S., Yoon H.J., Kim K.H., Hong Y.J., Park H.W., Kim J.H., Jeong M.H., Cho J.G., Park J.C., Kang J.C. Is myocardial infarction in patients without significant stenosis on a coronary angiogram as benign as believed? Chonnam Med J 2012; 48(1): 39-46, http://dx.doi. org/10.4068/cmj.2012.48.1.39.

11. Ishemicheskoe remodelirovanie levogo zheludochka [Ischemic remodeling of the left ventricle]. Pod red. Bokeriya L.A., Buziashvili Yu.I., Klyuchnikoval.V. [Bokeriya L.A., Buziashvili Yu.I., Klyuchnikov I.V. (editors)]. Moscow: Izdatel'stvo NTsSSKh im. A.N. Bakuleva RAMN; 2002.

12. Sevimli S., Büyükkaya E., Gündoğdu F., et al. Left ventricular function in patients with coronary slow flow: a tissue Doppler study. Arch Turk Soc Cardiol 2007; 35(6): 360-365.

13. Omidi N., Kashani B.S., Piranfar M.A., Khorgami M.R., Yekta B.G., Omidi H. The correlation of diastolic dysfunction with TIMI frame count in patients with chronic stable angina pectoris. Tehran Univ Med J 2012; 70(9): 555-63.

14. Nagueh S.F., Appleton C.P., Gillebert T.C., Marino P.N., Oh J.K., Smiseth O.A., Waggoner A.D., Flachskampf F.A., Pellikka P.A., Evangelisa A. Recommendations for the evaluation 
of left ventricular diastolic function by echocardiography. Eur J Echocardiogr 2009; 10(2): 165-193, http://dx.doi.org/10.1093/ ejechocard/jep007.

15. Vasyuk Yu.A., Khadzegova A.B., Yushchuk E.N., Krikunov P.V., Shkol'nik E.L., Ivanova S.V. Osnovnye printsipy otsenki diastolicheskoy funktsii serdtsa po dannym tkanevoy dopplerografii i vektornogo analiza skorosti dvizheniya miokarda [Basic principles of cardiac diastolic function evaluation according to tissue Doppler imaging data and vector analysis of myocardial movement velocity]. Moscow: Anakharsis; 2007. 